\title{
Risk factors associated with intensive care unit (ICU) admission and in-hospital death among adults hospitalized with COVID-19: a two-center retrospective observational study in tertiary care hospitals
}

\author{
Azin Shayganfar ${ }^{1}$ (D) $\cdot$ Ramin Sami $^{2}$ (D) $\cdot$ Somayeh Sadeghi ${ }^{2} \cdot$ Mehrnegar Dehghan $^{3} \cdot$ Nilufar Khademi $^{3}$. \\ Reyhaneh Rikhtehgaran ${ }^{4}$ • Reza Basiratnia ${ }^{1} \cdot$ Felora Ferdosi $^{1}$. Somayeh Hajiahmadi ${ }^{1}$ (I)
}

Received: 20 November 2020 / Accepted: 19 January 2021 / Published online: 3 February 2021

(C) American Society of Emergency Radiology 2021, corrected publication 2021

\begin{abstract}
Background The COVID-19 pandemic is straining the health care systems worldwide. Therefore, health systems should make strategic shifts to ensure that limited resources provide the highest benefit for COVID-19 patients.

Objective This study aimed to describe the risk factors associated with poor in-hospital outcomes to help clinicians make better patient care decisions.

Material and methods This retrospective observational study enrolled 176 laboratory-confirmed COVID-19 patients. Demographic characteristics, clinical data, lymphocyte count, CT imaging findings on admission, and clinical outcomes were collected and compared. Two radiologists evaluated the distribution and CT features of the lesions and also scored the extent of lung involvement. The receiver operating characteristic (ROC) curve was used to determine the optimum cutoff point for possible effective variables on patients' outcomes. Multivariable logistic regression models were used to determine the risk factors associated with ICU admission and in-hospital death.

Result Thirty-eight (21.5\%) patients were either died or admitted to ICU from a total of 176 enrolled ones. The mean age of the patients was $57.5 \pm 16.1$ years (males: $61 \%$ ). The best cutoff point for predicting poor outcomes based on age, CT score, and $\mathrm{O}_{2}$ saturation was 60 years (sensitivity: 71\%, specificity: $62 \%$ ), 10.5 (sensitivity: $73 \%$, specificity: 58\%), and 90.5\% (sensitivity: $73 \%$, specificity: $59 \%$ ), respectively. CT score cutoff point was rounded to 11 since this score contains only integer numbers. Multivariable-adjusted regression models revealed that ages of $\geq 60$ years, CT score of $\geq 11$, and $\mathrm{O}_{2}$ saturation of $\leq 90.5 \%$ were associated with higher worse outcomes among study population (odds ratio (OR): 3.62, 95\%CI: 1.35-9.67, $P=0.019$; OR: 4.38, 95\%CI: $1.69-11.35, P=0.002$; and OR: 2.78, 95\%CI: $1.03-7.47$, $P=0.042$, respectively).

Conclusion The findings indicate that older age, higher CT score, and lower $\mathrm{O}_{2}$ saturation could be categorized as predictors of poor outcome among COVID-19-infected patients. Other studies are required to prove these associations.
\end{abstract}

Keywords COVID-19 · Coronavirus · Risk factor · Poor outcome

Somayeh Hajiahmadi sohajiahmadi@gmail.com

1 Department of Radiology, School of Medicine, Isfahan University of Medical Sciences, Hezarjerib Avenue, Isfahan, Iran

2 Department of Internal medicine, School of Medicine, Isfahan University of Medical Sciences, Isfahan, Iran

3 Isfahan University of Medical sciences, Isfahan, Iran

4 Department of Mathematical Sciences, Isfahan University of Technology, Isfahan, Iran

\section{Introduction}

COVID-19 is a novel coronavirus that was first reported in January 2020 as the causative organism of clusters of pneumonia in Wuhan, China, and quickly spread throughout the world [1]. According to the virus's high infectivity, the World Health Organization (WHO) announced its pandemic on March 11, 2020. The clinical spectrum of COVID-19 seems to be broad, including asymptomatic infection, mild upper respiratory tract illness, severe pneumonia, acute respiratory distress syndrome (ARDS), and even death [2]. 
The standard of reference for COVID-19 diagnosis is realtime polymerase chain reaction (RT-PCR), which can detect a viral nucleic acid. A person with a positive RT-PCR result regardless of clinical signs and symptoms is considered a confirmed case [3].

There is no definite consensus on the use of chest imaging for the management of suspected COVID-19 pneumonia. CXR is not sensitive to COVID-19 infection in the early disease course with mild symptoms [4].

Based on RSNA (Radiologic Society of North America) and STR (Society of Thoracic Radiology) consensus statement, a chest CT scan should not be performed for routine screening of suspected COVID-19 pneumonia; it should be reserved for hospitalized, symptomatic patients [5]. However, several studies have been performed on the relationship of parenchymal involvement in CT scan and the severity of clinical symptoms and patient outcome [6].

Nowadays, due to the limited number of ICU beds and ventilators and the increasing number of patients with COVID-19 infection, an important challenge for healthcare systems is to determine the prognostic factors that significantly influence the clinical outcome of the patients.

Some studies are indicating old age, the presence of comorbidities, higher serum levels of D-dimer, lactate dehydrogenase (LDH), and C-reactive protein (CRP) as predictors of severity and prognosis of COVID-19 [7, 8].

This study analyzed the demographic characteristics, clinical data, lymphocyte count, and imaging findings of admitted COVID-19 patients to describe these patients' clinical characteristics and outcomes.

\section{Materials and methods}

\section{Patients}

In this study, we retrospectively reviewed the patients hospitalized at two tertiary care hospitals (Al-Zahra Hospital and Noor Hospital) in Isfahan, located in the center of Iran. The admission criteria were according to guidelines published by the Iranian Ministry of Health for the management of patients with suspected COVID-19 pneumonia based on a combination of clinical, laboratory, and radiologic information [9]. The demographic data (age and gender), comorbidity (ischemic heart disease, chronic respiratory disease, etc.), the clinical symptoms, and lymphocyte count at the admission time were retrospectively collected from patients' records.

This study was approved by the ethical committee of the Radiology Department, Isfahan University of Medical Sciences, Isfahan, Iran, and with a waiver of informed consent.

\section{Clinical symptoms}

The patients' clinical data at the time of admission, including the history of cough, fever, dyspnea, the time of symptoms initiation, the respiratory rate, and $\mathrm{O}_{2}$ saturation, were extracted from patients' records.

According to WHO classifications, all patients were classified into mild and severe pneumonia based on their clinical data at admission. The presence of respiratory rate $>30$ breaths $/ \mathrm{min}$, severe respiratory distress, or $\mathrm{SpO}_{2} \leq 93 \%$ with fever or suspected respiratory infection was considered as severe pneumonia. Mild pneumonia was considered non-severe pneumonia and no need for supplemental oxygen [10].

\section{CT images}

All chest CT images were acquired using Phillips Scanner with the patients in a supine position and at full inspiration and without the injection of contrast. Two board-certificate radiologists reviewed the images (AS and $\mathrm{SH}$ ). In the disagreement between the two primary radiologists, a third radiologist adjudicated the final decision. The two radiologists were blinded to the clinical and laboratory findings.

The CT images were evaluated for the presence of the following: (1) pattern characteristics including ground-glass opacity, consolidation, centrilobular nodules, bronchial wall thickening, reticulation, and vascular enlargement; (2) distribution characteristics including craniocaudal distribution (upper lobe predominant, lower lobe predominant, none), transverse distribution (central predominant or peripheral predominant, none), scattered (focal, multifocal, diffuse), and region (unilateral, bilateral); (3) ancillary findings including lymphadenopathy and pleural effusion.

A semi-quantitative scoring system was used to evaluate the extent of lung parenchymal involvement. A total of 5 lobes in both lungs were evaluated. The lobes were individually weighted based on parenchymal involvement. If no involvement was revealed, 0 was assigned. The involvement of less than $5 \%, 5 \%$ to $25 \%, 26 \%$ to $49 \%, 50 \%$ to $75 \%$, and $\geq 75 \%$ was scored as 1 to 5 , respectively. The final score was calculated, and a total score of 0 (no involvement) to 25 (diffuse interstitial involvement) was assigned for each patient (Fig. 1) [11].

\section{Short-term outcome}

In-hospital death and the need for ICU care are considered poor short-term outcomes. The discharge criteria were the presence of improvement in chest X-ray findings and clinical symptoms, the absence of fever for at least three days, and $\mathrm{O}_{2}$ saturation $>93 \%$ without mechanical ventilation. 
Fig. 1 An 81-year-old man with positive RT-PCR; symptoms: severe; CT findings: ground-glass opacities in both central and peripheral zones of both lungs without craniocaudal predilection; CT score: 14 ; outcome: mechanical ventilation

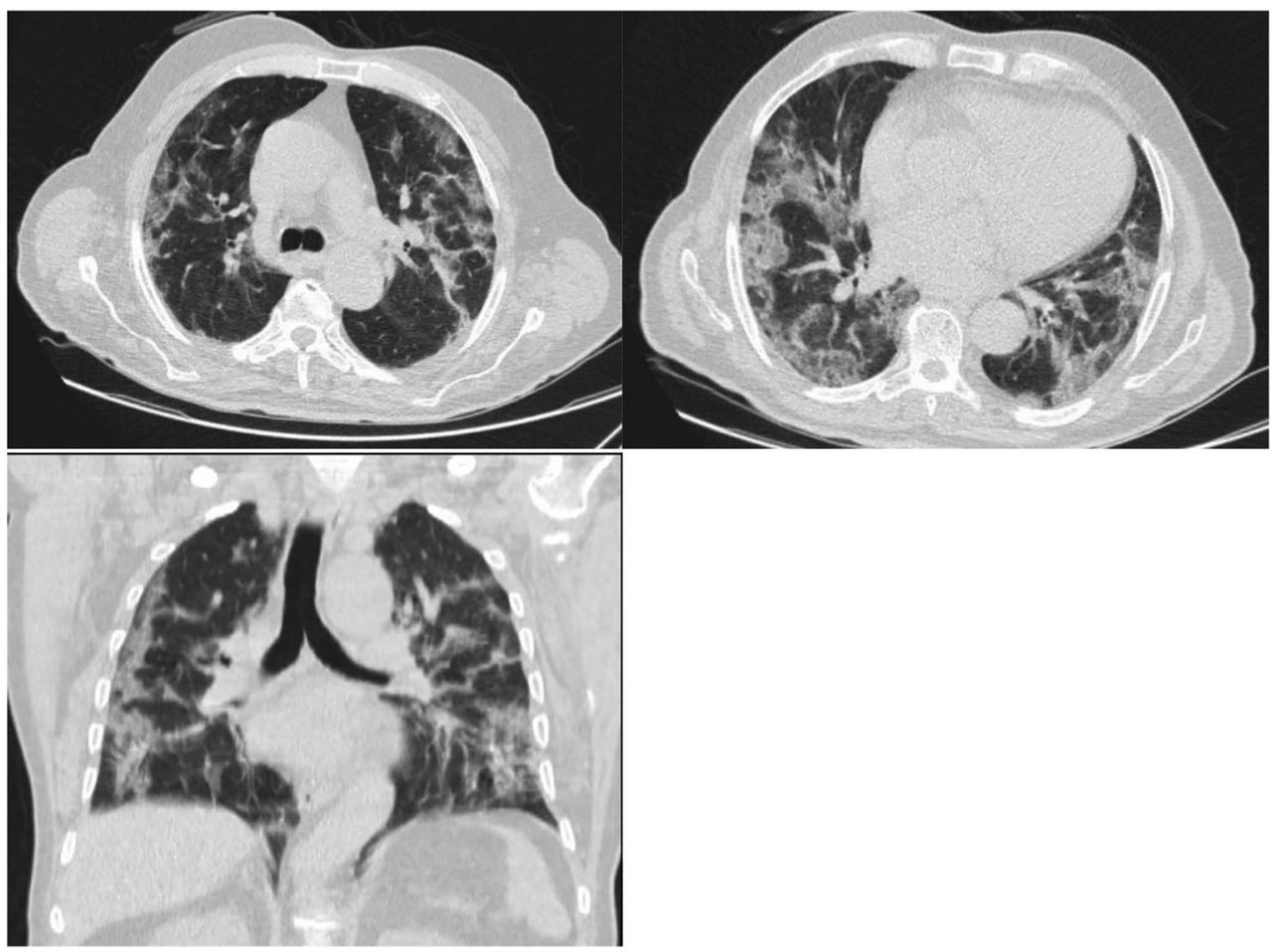

\section{Statistical analysis}

All the data were entered into the SPSS21 (SPSS Inc., Chicago, IL, USA). The quantitative data were reported using the mean value. Percentage and frequency were used to report the qualitative data. Independent sample $t$-test and chi-square tests were utilized used to examine the relation between numerical and nominal variables, respectively. The receiver operating characteristic (ROC) curve was used to determine the optimum cutoff point for possible effective variables on patients' outcomes. The relation between the pre-defined risk factors with the outcome was assessed with crude and adjusted models. In model 1, age and gender were adjusted. We further adjusted other confounding variables, including CT score, lymphocytes, $\mathrm{O}_{2}$ saturation, hypertension (HTN), diabetes mellitus (DM), chronic respiratory disease, cancer, chronic kidney disease (CKD), and hyperlipidemia (HLP) in the last model. The significance level was set at 0.05 .

\section{Results}

Of 176 recruited patients, $38(21.5 \%)$ subjects died or were admitted to ICU. Table 1 shows the characteristics of the study population according to discharge or ICU/death status. The study sample had a mean age of $57.5 \pm 16.1$ years, and $61 \%$ were male patients. Participants with poor outcomes had significantly higher means of age than the discharge group (67 \pm 13.2 years vs. $54.9 \pm 15.9$ years, $P<0.001)$. Those who died or were admitted to ICU had more prevalence of cardiovascular diseases (CVD) and lowered $\mathrm{O}_{2}$ saturation in comparison to discharged patients ( $36.8 \%$ vs. $14.5 \%, P=0.002$, and 85.21 $\pm 8.6 \%$ vs. $90.29 \pm 5.9, P<0.001)$. In terms of radiology findings and in comparison to patients with a good outcome, those with poor outcomes had significantly higher CT scores, higher bilateral lung involvement, but with lower consolidation rates $(14.39 \pm 5.8$ vs. $9.53 \pm 5.1, P<0.001,97.4 \%$ vs. $84.8 \%, P=0.038$, and 0 vs. $12.3 \%, P=0.023$ ). There was no significant association in terms of other radiologic findings between groups.

The area under the curve (AUC) for age based on the ROC curve was 0.703 (95\% confidence interval (CI): 0.611-0.794, $P<0.001$ ) (Fig. 2), and the best cutoff point for this variable was 60 years with the sensitivity of $71 \%$ and specificity of $62 \%$.

We also performed a ROC curve for the $\mathrm{CT}$ score and found that the AUC was 0.732 (95\%CI: $0.642-0.821, P<$ 0.001 ) (Fig. 3). The optimum cutoff value was set to be 10.5 (sensitivity: $73 \%$, specificity: 58\%). The cutoff point is rounded to the next integer number (11) since the CT score contains only integer numbers. The most common findings were mixed ground-glass opacity (ground-glass opacity and consolidation), which was seen among $82(46.6 \%)$ patients. The second most common finding was reticulation detected in 73 (41.5\%) patients. The most common distribution of pneumonia was peripherally $(61.4 \%)$, bilaterally $(87.5 \%)$, and multifocal (88.1\%) involvement. None of the patients had lymphadenopathy. Pleural effusion was detected in $9(5.1 \%)$ patients. 
Table 1 Baseline characteristics of the study population according to discharge or ICU/death status

\begin{tabular}{|c|c|c|c|c|c|}
\hline \multicolumn{2}{|l|}{ Variables } & \multirow{2}{*}{$\begin{array}{l}\text { Total }(n=176) \\
57.5 \pm 16.1\end{array}$} & \multirow{2}{*}{$\begin{array}{l}\begin{array}{l}\text { Discharge } \\
(n=138)\end{array} \\
54.9 \pm 15.9\end{array}$} & \multirow{2}{*}{$\begin{array}{l}\begin{array}{l}\text { ICU/death } \\
(n=38)\end{array} \\
67 \pm 13.2\end{array}$} & \multirow{2}{*}{$\begin{array}{l}P \\
<0.001\end{array}$} \\
\hline Age(years) & & & & & \\
\hline \multirow[t]{7}{*}{ Age group $(\%)$} & $20-30$ & $8(4.5)$ & $8(5.8)$ & 0 & \multirow[t]{7}{*}{0.001} \\
\hline & $30-40$ & $18(10.2)$ & $17(12.3)$ & $1(2.6)$ & \\
\hline & $40-50$ & $26(14.8)$ & $24(17.4)$ & $2(5.3)$ & \\
\hline & $50-60$ & $45(25.6)$ & $37(26.8)$ & $8(21.1)$ & \\
\hline & $60-70$ & $38(21.6)$ & $25(18.1)$ & $13(34.2)$ & \\
\hline & $70-80$ & $24(13.6)$ & $19(13.8)$ & $5(13.2)$ & \\
\hline & $80-90$ & $17(9.7)$ & $8(5.8)$ & $9(23.7)$ & \\
\hline \multirow{2}{*}{ Gender } & Males $(\%)$ & $108(61.4)$ & $81(58.7)$ & $27(71.1)$ & \multirow{2}{*}{0.166} \\
\hline & Females $(\%)$ & $68(38.6)$ & $57(41.3)$ & $11(28.9)$ & \\
\hline \multicolumn{6}{|l|}{ Previous medical history } \\
\hline \multicolumn{2}{|l|}{ HTN } & $54(30.7)$ & $43(31.2)$ & $11(28.9)$ & 0.793 \\
\hline \multicolumn{2}{|l|}{ Cardiovascular disease (\%) } & $34(19.3)$ & $20(14.5)$ & $14(36.8)$ & 0.002 \\
\hline \multicolumn{2}{|l|}{$\mathrm{DM}(\%)$} & $39(22.2)$ & $31(22.5)$ & $8(21.1)$ & 0.853 \\
\hline \multicolumn{2}{|l|}{ Chronic respiratory disease $(\%)$} & $16(9.1)$ & $12(8.7)$ & $4(10.4)$ & 0.728 \\
\hline \multicolumn{2}{|l|}{ Cancer $(\%)$} & $3(1.7)$ & $2(1.4)$ & $1(2.6)$ & 0.618 \\
\hline \multicolumn{2}{|l|}{ CKD $(\%)$} & $15(8.5)$ & $10(7.2)$ & $5(13.2)$ & 0.248 \\
\hline \multicolumn{2}{|l|}{$\operatorname{HLP}(\%)$} & $12(6.8)$ & $11(8)$ & $1(2.6)$ & 0.248 \\
\hline \multicolumn{6}{|l|}{ Clinical and laboratory data } \\
\hline \multicolumn{2}{|l|}{ Lymphocyte (\%) } & $1119.15 \pm 1418.8$ & $1179.05 \pm 1579.3$ & $893.26 \pm 389.2$ & 0.291 \\
\hline \multicolumn{2}{|l|}{$\mathrm{O}_{2}$ saturation $(\%)$} & $89.19 \pm 6.9$ & $90.29 \pm 5.9$ & $85.21 \pm 8.6$ & $<0.001$ \\
\hline \multicolumn{2}{|l|}{ Respiratory rates (rates/minute) } & $21.42 \pm 5.1$ & $21.13 \pm 5.1$ & $22.44 \pm 5$ & 0.162 \\
\hline \multicolumn{6}{|l|}{ CT findings } \\
\hline \multicolumn{2}{|l|}{ CT score } & $10.58 \pm 5.6$ & $9.53 \pm 5.1$ & $14.39 \pm 5.8$ & $<0.001$ \\
\hline \multicolumn{2}{|l|}{ Ground-glass opacity (\%) } & $70(39.8)$ & $51(37)$ & $19(50)$ & 0.146 \\
\hline Mixed ground-glass opacity and c & ation $(\%)$ & $82(46.6)$ & $64(46.4)$ & $18(47.4)$ & 0.914 \\
\hline Consolidation $(\%)$ & & $17(9.7)$ & $17(12.3)$ & 0 & 0.023 \\
\hline Centrilobular nodules (\%) & & $8(4.5)$ & $7(5.1)$ & $1(2.6)$ & 0.522 \\
\hline Bronchial wall thickening (\%) & & $14(8)$ & $10(7.2)$ & $4(10.5)$ & 0.508 \\
\hline Reticulation (\%) & & $73(41.5)$ & $57(41.3)$ & $16(42.1)$ & 0.929 \\
\hline Vascular enlargement (\%) & & $13(7.4)$ & $11(8)$ & $2(5.3)$ & 0.572 \\
\hline Pleural effusion (\%) & & $9(5.1)$ & $8(5.8)$ & $1(2.6)$ & 0.433 \\
\hline Upper or lower involvement (\%) & Upper & $5(2.8)$ & $5(3.6)$ & 0 & 0.101 \\
\hline & Lower & $89(50.6)$ & $74(53.6)$ & $15(39.5)$ & \\
\hline & None & $82(46.6)$ & $59(42.8)$ & $23(60.5)$ & \\
\hline Unilateral/bilateral (\%) & Unilateral & $22(12.5)$ & $21(15.2)$ & $1(2.6)$ & 0.038 \\
\hline & Bilateral & $154(87.5)$ & $117(84.8)$ & $37(97.4)$ & \\
\hline Central/peripheral/none $(\%)$ & Central & $9(5.1)$ & $7(5.1)$ & $2(5.3)$ & 0.880 \\
\hline & Peripheral & $108(61.4)$ & $86(62.3)$ & $22(57.9)$ & \\
\hline & None & $59(33.5)$ & $45(32.6)$ & $14(36.8)$ & \\
\hline Focal/multifocal/diffuse (\%) & Focal & $17(9.7)$ & $16(11.6)$ & $1(2.6)$ & 0.107 \\
\hline & Multifocal & $155(88.1)$ & $120(87)$ & $35(92.1)$ & \\
\hline & Diffuse & $4(2.3)$ & $2(1.4)$ & $2(5.3)$ & \\
\hline
\end{tabular}

Also, no difference between discharge or ICU/death status was seen regarding lymphopenia lymphocyte count $(p=0.291)$.

Finally, we calculated the AUC for $\mathrm{O}_{2}$ saturation and the result was 0.713 (95\%CI: $0.618-0.808, P<0.001)$ (Fig. 4) with the best cutoff point of $90.5 \%$ (sensitivity: $73 \%$, specificity: $59 \%$ ).

Table 2 presents the multivariable-adjusted odds ratio of death/ICU status according to age, CT score, and $\mathrm{O}_{2}$ 


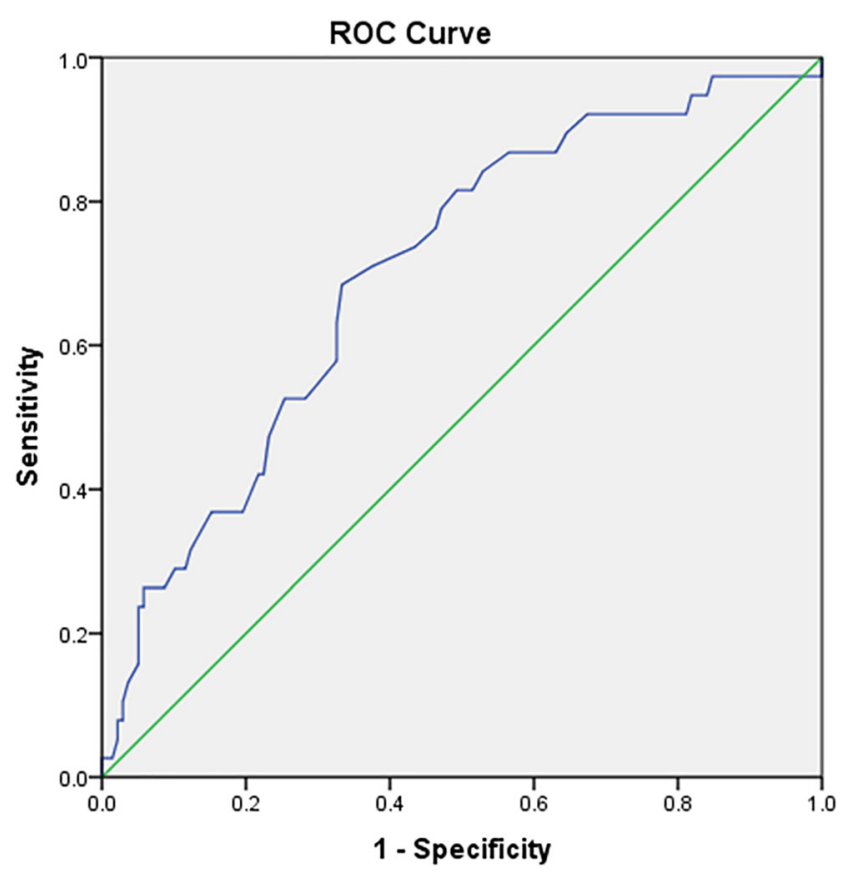

Diagonal segments are produced by ties.

Fig. 2 ROC curve based on age among the study population

saturation. In comparison to patients with less than 60 years, those with higher ages had a significantly higher likelihood of poor outcome both in crude and adjusted models (crude: 4.05 (95\%CI: $1.85-8.86, P<0.001$ ), model 1: 3.95 (95\%CI: 1.80 8.66, $P=0.001$ ), model 2: 3.62 (95\%CI: $1.35-9.67, P=$ $0.019)$ ). Patients with higher CT scores had 3.86 (95\% CI: 1.74-8.57, $P=0.001)$ times higher risks of poor outcomes

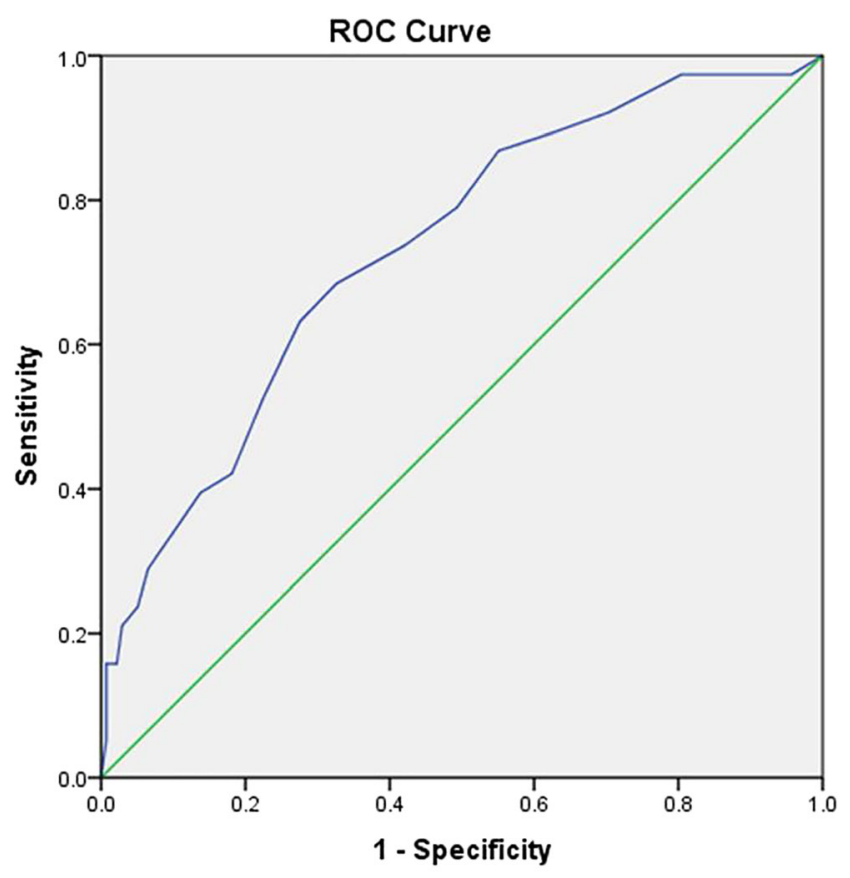

Diagonal segments are produced by ties.

Fig. 3 ROC curve based on CT score among the study population

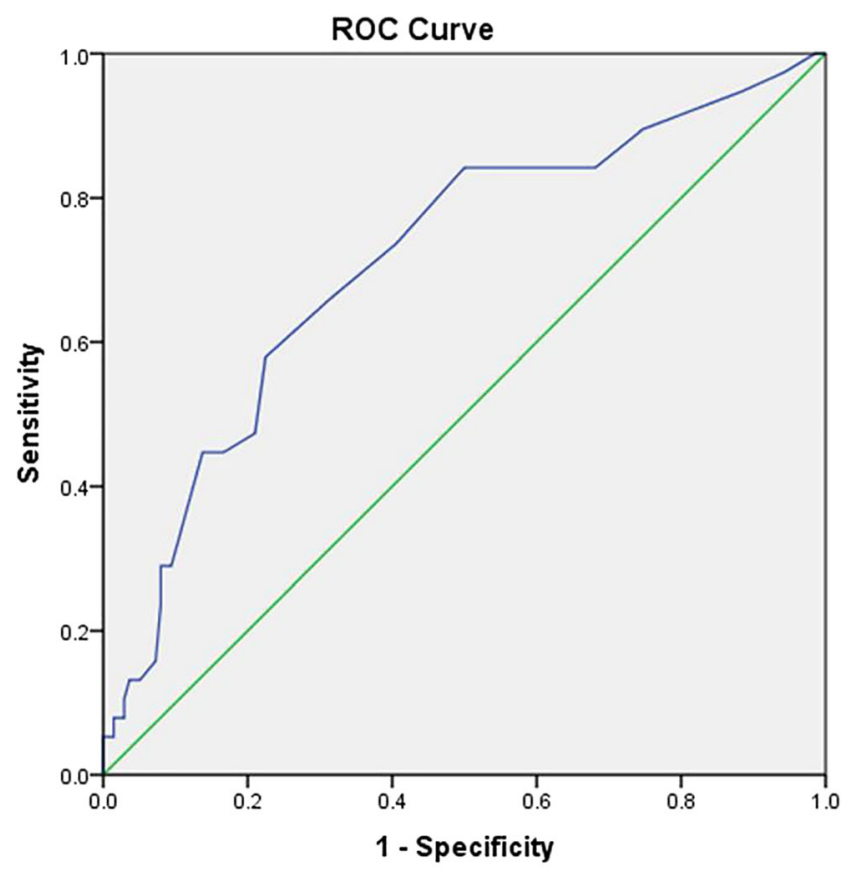

Diagonal segments are produced by ties.

Fig. 4 ROC curve based on $\mathrm{O}_{2}$ saturation among the study population

than the reference group in the crude model. This higher likelihood remained significant after adjusting all potential confounders (OR: 4.38, 95\% CI: 1.69-11.35, $P=0.002$ ). In terms of $\mathrm{O}_{2}$ saturation, patients with the saturation of less than 90.5\% had 4.10 (95\%CI: $1.84-9.10, P=0.001$ ) and 2.78 (95\%CI: $1.03-7.47, P=0.042$ ) times higher odds of worse clinical outcome compared to those with higher $\mathrm{O}_{2}$ saturation in the crude and adjusted model, respectively.

\section{Discussion}

This study evaluated the clinical data, lymphocyte count, and chest $\mathrm{CT}$ findings of 176 patients with a positive RT-PCR for COVID-19 to determine significant prognostic factors for short-term outcomes.

We found that among a sample of adults hospitalized with laboratory-confirmed COVID-19, almost one-fifth required ICU admission or died during hospitalization. The most prevalent comorbidities were HTN (30.7\%), DM (22.2\%), and CVD (19.3\%), respectively. Older age, higher CT score, and lower $\mathrm{O}_{2}$ saturation were associated with higher ICU admission risks and in-hospital mortality.

ICU admissions and death due to COVID-19 have been shown to occur more often with increasing age $[12,13]$. In our study, the age of $\geq 60$ years was the strongest independent predictor of ICU admission and in-hospital mortality. These findings are in concordance with other studies from China, Europe, and the USA [13-17]. Our findings showed that older patients are particularly vulnerable to worse outcomes from 
Table 2 The multivariable-adjusted odds ratio of death/ICU status according to age, CT score, and $\mathrm{O}_{2}$ saturation

\begin{tabular}{|c|c|c|c|c|c|c|c|c|c|}
\hline \multirow[t]{2}{*}{ Models } & \multicolumn{2}{|l|}{ Age } & \multirow[t]{2}{*}{$P$} & \multicolumn{2}{|c|}{ CT score } & \multirow[t]{2}{*}{$P$} & \multicolumn{2}{|c|}{$\mathrm{O}_{2}$ saturation } & \multirow[t]{2}{*}{$P$} \\
\hline & $<60$ & $\geq 60$ & & $<10.5$ & $\geq 10.5$ & & $>90.5$ & $\leq 90.5$ & \\
\hline Crude & 1.00 & $4.05(1.85-8.86)$ & $<0.001$ & 1.00 & $3.86(1.74-8.57)$ & 0.001 & 1.00 & $4.10(1.84-9.10)$ & 0.001 \\
\hline Model $1 *$ & 1.00 & $3.95(1.80-8.66)$ & 0.001 & 1.00 & $3.69(1.61-8.46)$ & 0.002 & 1.00 & $2.99(1.28-6.95)$ & 0.011 \\
\hline Model 2** & 1.00 & $3.62(1.35-9.67)$ & 0.019 & 1.00 & $4.38(1.69-11.35)$ & 0.002 & 1.00 & $2.78(1.03-7.47)$ & 0.042 \\
\hline
\end{tabular}

*Adjusted for age and sex

**Adjusted for age, sex, CT score, lymphocytes, $\mathrm{O}_{2}$ saturation, HTN, DM, COPD, cancer, CKD, HLP with the exception of the desired variable

COVID-19 disease and should be targeted for aggressive preventive measures [18].

We showed that the extent of lung parenchymal involvement in chest CT was associated with the disease's severity. In terms of radiology findings, those patients with poor outcomes had significantly higher $\mathrm{CT}$ scores, higher bilateral lung involvement, but with lower consolidation rates $(P<0.001, P=0.038$, and $P=$ 0.023 , respectively). The results are in concordance with the results reported by Li et al., suggesting the quantitative CT image analysis as an accurate method for clinical severity assessment and associated with poor outcome [19]. Another study done on 101 patients with COVID-19 reported that the extension of parenchymal lung involvement was significantly higher in patients with severe and fatal disease types [20].

We found an association between CVD and poor shortterm outcomes; however, we cannot find any significant association between DM, CKD, HTN, or chronic respiratory disease, and ICU admission or in-hospital mortality. In this regard, Kim et al., in their study on the US population, showed an association between DM and CKD with ICU admission. They also cannot found any association between HTN and poor outcomes [21]. Like our study, Killerby et al., in another study on the US population, reported no significant association between HTN and poor outcomes; however, they showed that HTN was a more prevalent comorbidity in the US population. [22]. On the other hand, several Chinese studies reported that HTN to be associated with more severe clinical outcomes $[17,23,24]$. Further studies are needed to determine whether HTN, which is highly prevalent in the admitted COVID-19 patients, increases the risk of in-hospital complications in these patients. Similarly, the associations between the duration and degree of blood pressure control in HTN and outcomes of COVID-19 disease require further investigation.

In this study, we also assessed the role of clinical characteristics, lymphocyte count, and CT imaging features in predicting the patients' short-term prognosis. The results indicated that $\mathrm{O}_{2}$ saturation $\leq 90.5 \%$, age $\geq 60$, and CT score $\geq 11$ were associated with increased odds of ICU admission or in-hospital death. Because COVID-19 primarily affects the respiratory system, markers that reflect respiratory function would more likely relate to outcomes. Therefore, systemic oxygenation is a potential prognostic biomarker. On the other hand, hypoxemia was associated with an increase of inflammatory biomarkers (higher D-dimer levels, WBC counts, neutrophil counts, and CRP levels) and acute inflammation of the respiratory system [25].

Davarpanah et al. showed that older age, $\mathrm{SpO}_{2}<88 \%$, and higher chest $\mathrm{CT}$ scores were significant predictors for ICU admission or in-hospital death [9]. In another study by $\mathrm{Li}$ et al., chest CT score $>7$ and age $>50$ years indicated critical COVID-19 pneumonia [26]. Tan et al. suggested that lymphopenia could be considered a useful indicator of severe COVID-19 disease [27]. However, we do not find a significant association between poor in-hospital outcomes and lymphopenia. In a study by Xu et al., $\geq 2$ comorbidities, leukocytosis, lymphopenia, chest CT score $>14$, and older age were associated with increased odds of poor outcome [28].

Altogether, due to the rapid growth of critically ill COVID-19 patients and limited health resources, especially ICU beds, we need effective triage strategies for identifying patients at higher risk for poor outcomes to achieve effective resource allocation.

This study's limitations were the retrospective nature of the study and the relatively small sample size. Additionally, it is probably that not all COVID-19-associated hospitalizations were captured because of the lack of widespread testing capability during the study period and because the recognition of COVID-19 patients was mostly dependent on clinician directed testing. Finally, due to the study's retrospective nature, we did not record the time between the onset of symptoms and the CT examination date.

\section{Conclusion}

We found that older age, higher CT severity score, and hypoxemia were risk factors for poor in-hospital outcomes in COVID-19 PCR-positive patients. Further studies are required to prove these associations.

Availability of data and material All data will be made available based on reasonable request. 
Author contributions All authors contributed to the study conception and design. Material preparation, data collection, and data analysis were performed by A. Shayganfar, S. Hajiahmadi, R. Sami, S. Sadeghi, M. Dehghan, N. Khademi, R. Basiratnia, and F. Ferdosi. The first draft of the manuscript was written by S. Hajiahmadi and supervised by A. Shayganfar. Statistics analysis was performed by R. Rikhtegaran. All authors commented on previous versions of the manuscript. All authors read and approved the final manuscript.

\section{Declarations}

\section{Ethical approval IR.MUI.MED.REC.1398.720.}

Consent to participate A waiver of informed consent was granted for this retrospective study.

Consent for Publication The included CT images are not necessary to obtain consent as without any identifying information.

Conflict of interest The authors declare that they have no conflict of interest.

\section{References}

1. Xu X, Chen P, Wang J, Feng J, Zhou H, Li X et al (2020) Evolution of the novel coronavirus from the ongoing Wuhan outbreak and modeling of its spike protein for risk of human transmission. Sci China Life Sci 63(3):457-460

2. Huang C, Wang Y, Li X, Ren L, Zhao J, Hu Y et al (2020) Clinical features of patients infected with 2019 novel coronavirus in Wuhan, China. Lancet 395(10223):497-506

3. Organization WH (2020) Laboratory testing for coronavirus disease 2019 (COVID-19) in suspected human cases: interim guidance, 2 March 2020. World Health Organization

4. Wong HYF, Lam HYS, Fong AH-T, Leung ST, Chin TW-Y, Lo CSY et al (2020) Frequency and distribution of chest radiographic findings in COVID-19 positive patients. Radiology. 201160

5. Simpson S, Kay FU, Abbara S, Bhalla S, Chung JH, Chung M et al (2020) Radiological Society of North America Expert Consensus Statement on Reporting Chest CT Findings Related to COVID-19. Endorsed by the Society of Thoracic Radiology, the American College of Radiology, and RSNA. Radiology: Cardiothoracic Imaging 2(2):e200152

6. Francone M, Iafrate F, Masci GM, Coco S, Cilia F, Manganaro L et al (2020) Chest CT score in COVID-19 patients: correlation with disease severity and short-term prognosis. Eur Radiol:1-10

7. Zhao X, Zhang B, Li P, Ma C, Gu J, Hou P et al (2020) Incidence, clinical characteristics and prognostic factor of patients with COVID-19: a systematic review and meta-analysis. MedRxiv

8. Zhou F, Yu T, Du R, Fan G, Liu Y, Liu Z et al (2020) Clinical course and risk factors for mortality of adult inpatients with COVID-19 in Wuhan, China: a retrospective cohort study. Lancet

9. Davarpanah AH, Asgari R, Moharamzad Y, Mahdavi A, Abrishami A, Nekooghadam S et al (2020) Risk factors for poor outcome in patients with severe viral pneumonia on chest CT during the COVID-19 outbreak: a perspective from Iran. SN comprehensive clinical medicine 2(9):1366-1376

10. Organization WH (2020) Clinical management of severe acute respiratory infection (SARI) when COVID-19 disease is suspected: interim guidance, 13 March 2020. World Health Organization
11. Pan F, Ye T, Sun P, Gui S, Liang B, Li L et al (2020) Time course of lung changes on chest CT during recovery from 2019 novel coronavirus (COVID-19) pneumonia. In: Radiology

12. Guan W-J, Ni Z-Y, Hu Y, Liang W-H, Ou C-Q, He J-X, et al. Clinical characteristics of coronavirus disease 2019 in China. $\mathrm{N}$ Engl J Med 2020;382(18):1708-1720.

13. COVID TC, Team R (2020) Severe outcomes among patients with coronavirus disease 2019 (COVID-19)-United States, February 12March 16, 2020. MMWR Morb Mortal Wkly Rep 69(12):343-346

14. Petrilli CM, Jones SA, Yang J, Rajagopalan H, O'Donnell L, Chernyak $\mathrm{Y}$ et al (2020) Factors associated with hospital admission and critical illness among 5279 people with coronavirus disease 2019 in New York City: prospective cohort study. bmj 369

15. Chen T, Wu D, Chen H, Yan W, Yang D, Chen G et al (2020) Clinical characteristics of 113 deceased patients with coronavirus disease 2019: retrospective study. Bmj. 368

16. Onder G, Rezza G, Brusaferro S (2020) Case-fatality rate and characteristics of patients dying in relation to COVID-19 in Italy. Jama. 323(18):1775-1776

17. Wu C, Chen X, Cai Y, Zhou X, Xu S, Huang H et al (2020) Risk factors associated with acute respiratory distress syndrome and death in patients with coronavirus disease 2019 pneumonia in Wuhan, China. JAMA Intern Med

18. Chow N, Fleming-Dutra K, Gierke R, Hall A, Hughes M, Pilishvili $T$ et al (2020) Preliminary estimates of the prevalence of selected underlying health conditions among patients with coronavirus disease 2019-United States. February 12-March 28:2020

19. Li K, Fang Y, Li W, Pan C, Qin P, Zhong Y et al (2020) CT image visual quantitative evaluation and clinical classification of coronavirus disease (COVID-19). Eur Radiol:1-10

20. Zhao W, Zhong Z, Xie X, Yu Q, Liu J (2020) Relation between chest CT findings and clinical conditions of coronavirus disease (COVID-19) pneumonia: a multicenter study. Am J Roentgenol 214(5):1072-1077

21. Kim L, Garg S, O'Halloran A, Whitaker M, Pham H, Anderson EJ et al (2020) Risk factors for intensive care unit admission and inhospital mortality among hospitalized adults identified through the US coronavirus disease 2019 (COVID-19)-associated hospitalization surveillance network (COVID-NET). Clin Infect Dis

22. Killerby ME, Link-Gelles R, Haight SC, Schrodt CA, England L, Gomes DJ et al (2020) Characteristics associated with hospitalization among patients with COVID-19-Metropolitan Atlanta, Georgia, March-April 2020. Morb Mortal Wkly Rep 69(25):790

23. Guan W-J, Liang W-H, Zhao Y, Liang H-R, Chen Z-S, Li Y-M et al (2020) Comorbidity and its impact on 1590 patients with Covid-19 in China: A Nationwide Analysis. Eur Respir J 55(5)

24. Wang K, Zuo P, Liu Y, Zhang M, Zhao X, Xie S et al (2020) Clinical and laboratory predictors of in-hospital mortality in patients with COVID-19: a cohort study in Wuhan, China. Clin Infect Dis

25. Xie J, Covassin N, Fan Z, Singh P, Gao W, Li G et al (2020) Association between hypoxemia and mortality in patients with COVID-19. In: Mayo Clinic Proceedings. Elsevier

26. Li K, Wu J, Wu F, Guo D, Chen L, Fang Z et al (2020) The clinical and chest CT features associated with severe and critical COVID19 pneumonia. In: Investigative radiology

27. Tan L, Wang Q, Zhang D, Ding J, Huang Q, Tang Y-Q et al (2020) Lymphopenia predicts disease severity of COVID-19: a descriptive and predictive study. Signal transduction and targeted therapy 5(1):1-3

28. Xu PP, Tian RH, Luo S, Zu ZY, Fan B, Wang XM et al (2020) Risk factors for adverse clinical outcomes with COVID-19 in China: a multicenter, retrospective, observational study. Theranostics. 10(14):6372

Publisher's note Springer Nature remains neutral with regard to jurisdictional claims in published maps and institutional affiliations. 